\title{
Liquid-liquid two-phase flow patterns in ultra-shallow straight and serpentine microchannels
}

\author{
Zan $\mathrm{Wu}^{1}$ (D) $\cdot$ Bengt Sundén ${ }^{1}$
}

Received: 19 February 2018 / Accepted: 29 September 2018 / Published online: 6 October 2018

(C) The Author(s) 2018

\begin{abstract}

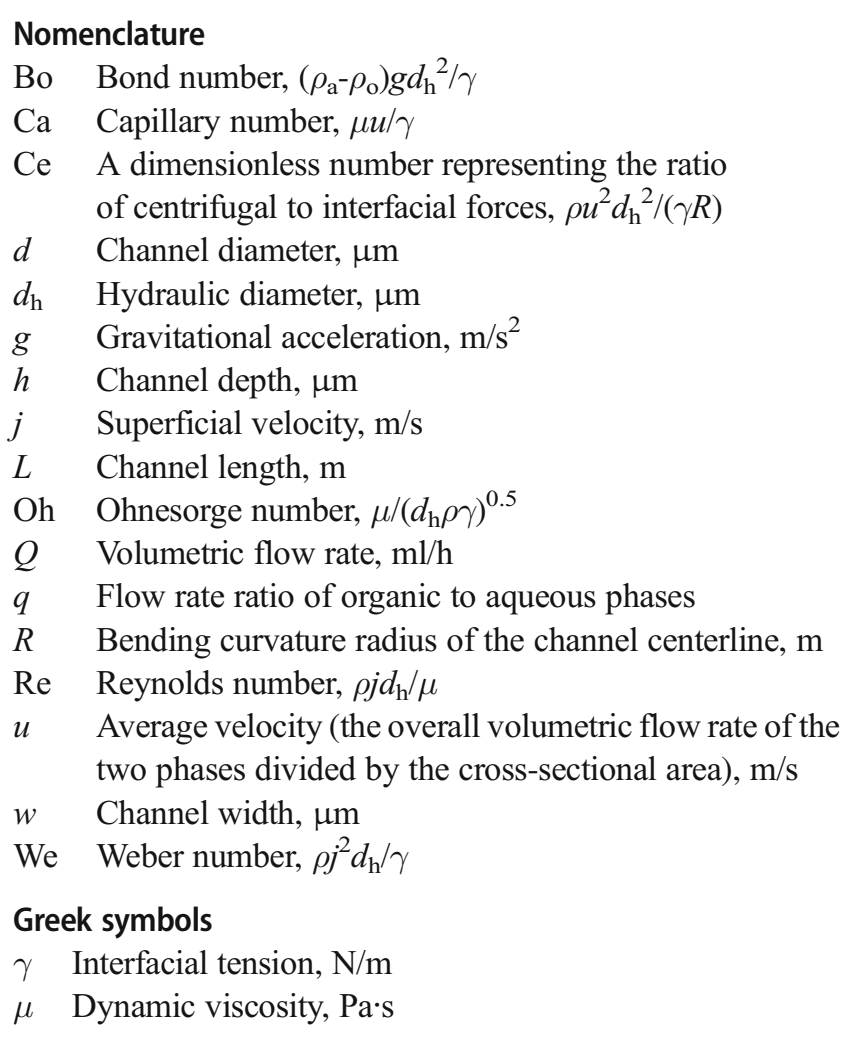

Water-butanol and water-hexane flows were visualized in ultra-shallow straight and serpentine microchannels with a crossjunction. At the inlet cross-junction, three major flow patterns including tubing/threading, dripping and jetting were mapped using the aqueous Capillary number versus the organic Weber number. Correspondingly, in the main microchannel, annular flow, slug flow and droplet flow were mapped using combined dimensionless numbers (Weber number times Ohnesorge number) of both phases. The flow pattern transitions were explained based on a force analysis, considering the phase flow rates, junction angle between the side feeding channels and the central feeding channel as well as aspect ratios. Compared to the straight microchannel, the dripping regime at the inlet junction and the slug flow occupy larger zones in serpentine microchannels because the centrifugal force tends to break up the organic annular core into slugs and droplets over the bends.

Zan $\mathrm{Wu}$

zan.wu@energy.lth.se

1 Department of Energy Sciences, Lund University, Box 118, SE-22100 Lund, Sweden

\author{
$\rho \quad$ Density, $\mathrm{kg} / \mathrm{m}^{3}$ \\ Subscript \\ ave Average \\ a Aqueous phase \\ c Continuous phase \\ d Dispersed phase \\ o Organic phase
}

\section{Introduction}

Concerning process intensification and scale miniaturization, micro-structured devices (e.g., microscale heat exchangers, microreactors, micromixers) are widely used because they possess merits such as high heat and mass transport rates, enhanced mixing, fast reaction, energy and raw material savings, and easiness of numbering up [1]. Two-phase microstructured systems are significant in extraction, emulsification, polymerization, nitration, nanoparticle synthesis, phasetransfer catalysis, pharmaceutical and biochemistry etc. Twophase flow patterns at microscale are not much covered, e.g., refs. [2-6] for gas-liquid flow, and refs. [7-12] for liquidliquid flow. Inlet microchannels intersect at junctions which define local flow fields causing interface deformation. A cross-junction, as a hydrodynamic focusing geometry, consists of three microchannels that intersect at a certain angle, with one phase from two side inlets and with the other from the central inlet $[11,13]$. The cross-junction creates approximately an extensional flow, a common feature of flow- 
focusing geometries [14]. Various flow patterns occur when two phases meet at the junction. For example, Cubaud and Mason [15] observed tubing, threading, displacement, dripping and jetting flow regimes for various immiscible fluid pairs in a cross-junction of straight microchannels with square cross sections, covering more than an order of magnitude of difference in viscosity ratios and interfacial tensions. Squeezing, dripping and jetting were realized by De Menech et al. [16] to form droplets for microfluidic T-junctions. Fu et al. [17] identified viscous displacement, tubing, dripping and jetting in a microfluidic cross-junction for silicone oilwater flow. Recently, Wu et al. [11] mapped flow patterns at cross-junctions and studied slug hydrodynamics for five liquid-liquid systems flowing in three square glass microchannels of channel depths from $200 \mu \mathrm{m}$ to $600 \mu \mathrm{m}$.

Besides the flow regimes at the inlet junction, flow regimes downstream in the main microchannel are of equal importance for transport processes and reactions. For flow patterns captured at downstream locations "far" from the inlet, a different flow-pattern terminology was used, e.g., annular, slug and droplet. The same flow pattern captured at the inlet junction may evolve along the downstream microchannel into different flow patterns [5, 11]. For example, as stated in Wu et al. [11], the tubing/threading pattern at the cross-junction either evolves into an annular flow or a slug flow at downstream locations in the main microchannel; the dripping regime at the cross-junction either develops into a slug flow or a droplet flow at downstream axial locations. Slug hydrodynamics (e.g., slug size and velocity) and their dependence on $\mathrm{Ca}$ and $q$ have been analyzed [14, 17-19].

Few works are devoted to serpentine or meandering microchannels even if such an arrangement is preferable as a mixer or a reactor for a long flow path on a small chip and for secondary flows to enhance mixing [20-26]. Bends and curved channels affect single-phase and two-phase flows differently [24]. For one-phase flow through a bend, there are two counter-rotating Dean vortices within the microchannel cross section [27]. For two-phase flow, the centrifugal force generates two different sized vortices providing a threedimensional (3D) asymmetrical recirculation in serpentine and meandering microchannels [28]. The asymmetrical motion intensifies as the centrifugal force increases by reducing the bend curvature radius or by increasing the flow rates. Table 1 provides a brief summary of relevant works on twophase flows in serpentine/meandering micro-/mini-channels. Among them, most of the reported works considered gasliquid flows; works focused on liquid-liquid flows are very limited. Besides, the works focused on channels of aspect ratios $<2.7$. In this work, the aspect ratio equals the channel width over its depth.

As flow structures are closely coupled with heat and mass transport processes $[2,11]$, this study intends to experimentally investigate liquid-liquid flow patterns both at/near the

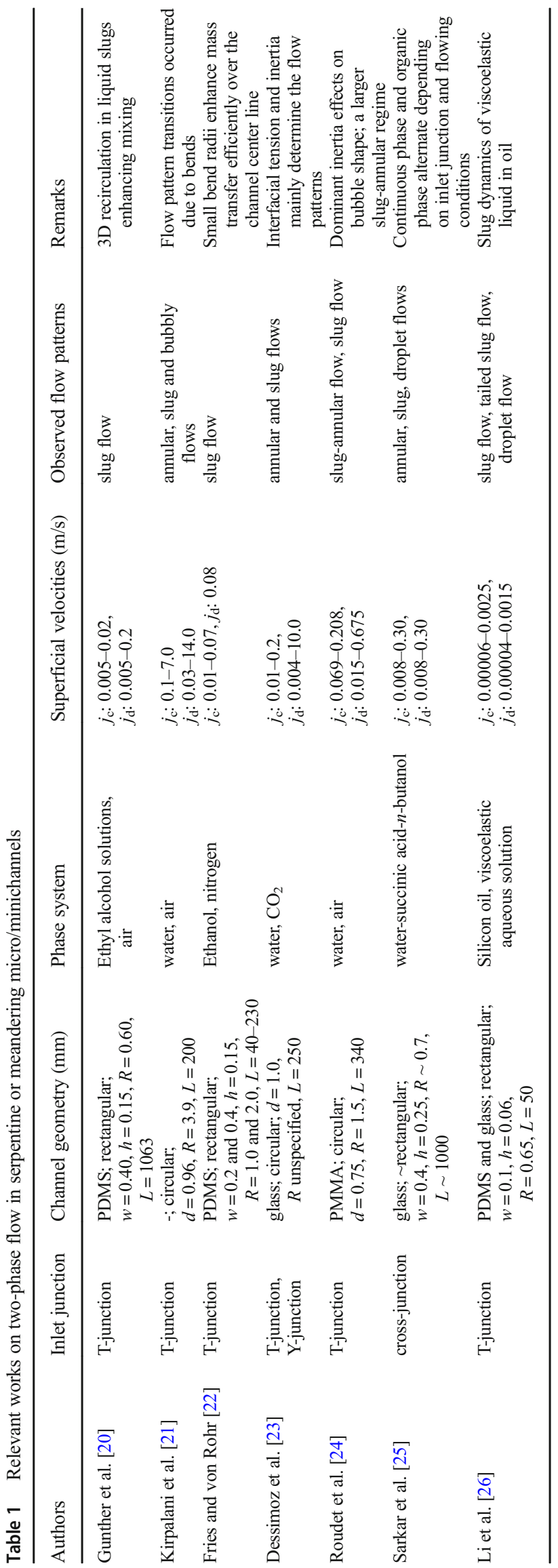


Fig. 1 Drawing of the test rig

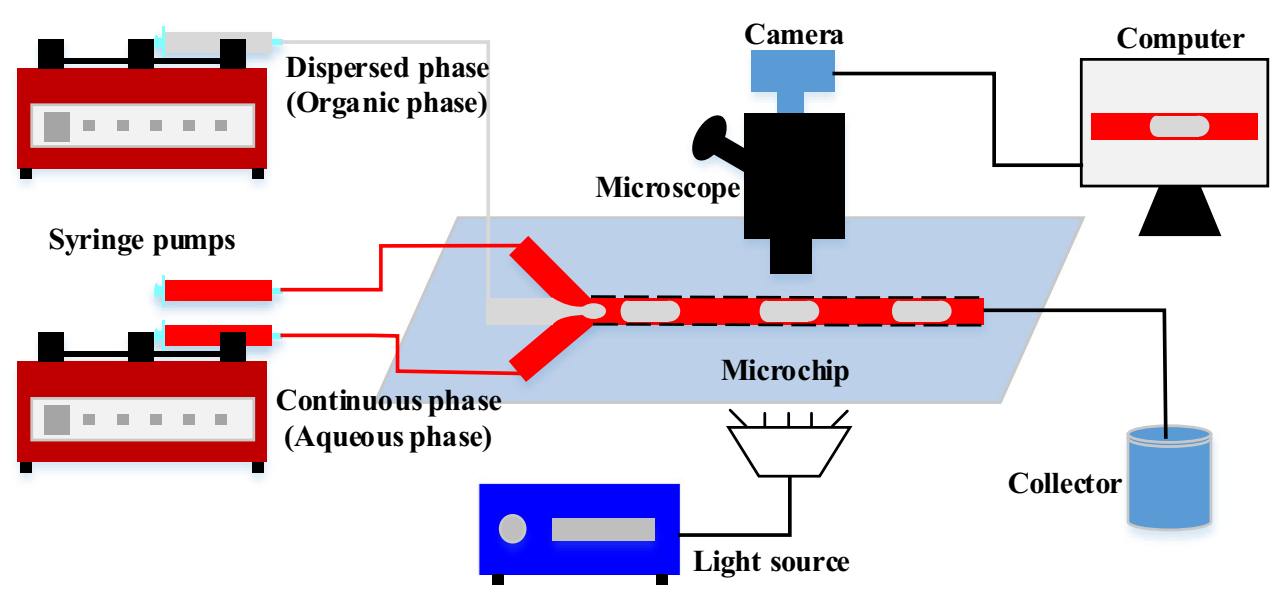

cross-junction and at downstream locations "far" from the cross-junction in the main microchannel of quasi-trapezoidal straight and serpentine microchannels with an aspect ratio of 5.3 by employing water-butanol and water-hexane systems.

\section{Experimental setup and procedure}

As seen in Fig. 1, organic solvents and water were delivered to microchannels and controlled by two syringe pumps (New Era). Test sections (straight and serpentine microchannels) were placed horizontally. A stereo microscope (Motic) and a camera (Olympus) were used to capture the flow patterns inside the microchannels. Two quasi-trapezoidal microchannels, one straight and one serpentine, fabricated by isotropic wet etching in glass with a glass lid bonded on the top, were used as test sections with excellent chemical resistance. The straight and serpentine microchannel layouts are shown in Fig. 2. The inlet and outlet junctions of the two microchannels are crossjunctions, with an angle of $45^{\circ}$ between the side channel and the central channel. The length of the straight main microchannel is $50 \mathrm{~mm}$. For the serpentine microchannel, as
Fig. 2 Description of the configuration and the crosssection geometry of a the straight microchannel and $\mathbf{b}$ the serpentine microchannel

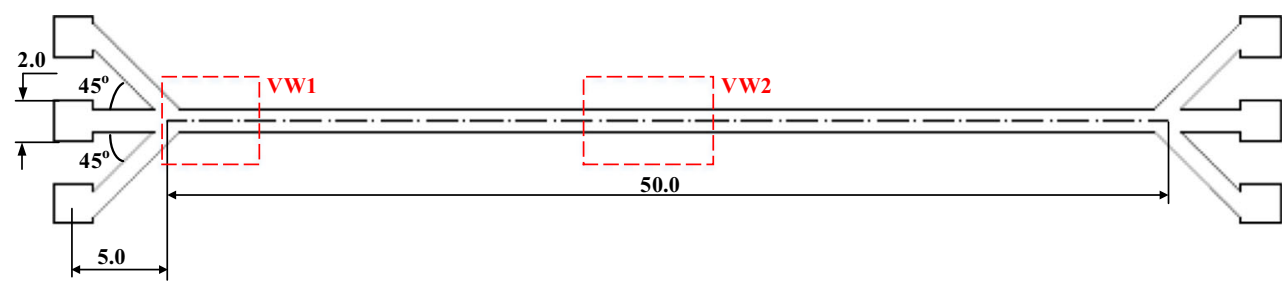

(a)

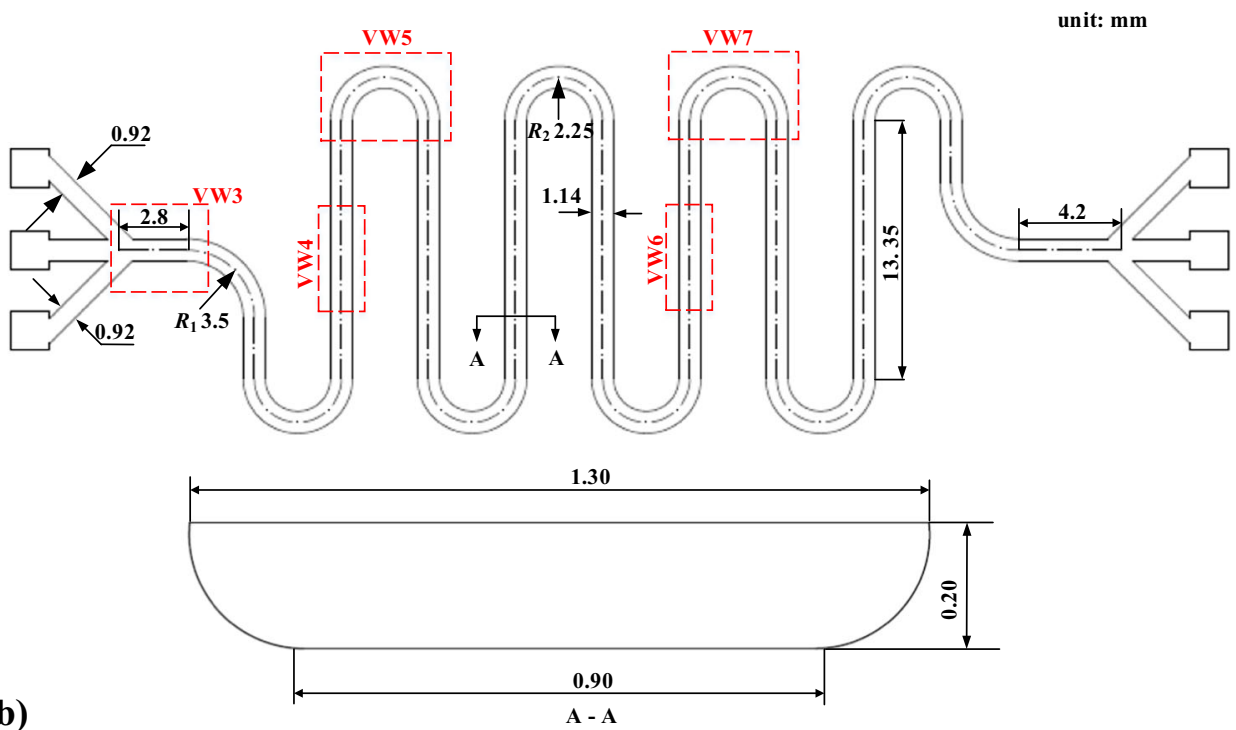


Table 2 Fluid properties at 20.0 $\pm 0.5^{\circ} \mathrm{C}$

\begin{tabular}{lllll}
\hline & Organic phase & Density, $\rho\left(\mathrm{kg} / \mathrm{m}^{3}\right)$ & Viscosity, $\mu(\mathrm{Pa} \cdot \mathrm{s})$ & Interfacial tension, $\gamma(\mathrm{N} / \mathrm{m})$ \\
\hline Water & & 998.2 & 0.001 & - \\
& & & & \\
& Butanol & 0.00294 & water-butanol: 0.0018 \\
& Hexane & 654.8 & 0.0003 & water-hexane: 0.051 \\
\hline
\end{tabular}

shown in Fig. 2b, there is a straight section of $2.8 \mathrm{~mm}$ between the inlet junction and the first $90^{\circ}$ bend, and a straight section of $4.2 \mathrm{~mm}$ between the last $90^{\circ}$ bend and the outlet junction. The two $90^{\circ}$ bends have a curvature radius of the channel centerline $\left(R_{1}\right)$ of $3.5 \mathrm{~mm}$, while the eight $180^{\circ}$ bends between the two $90^{\circ}$ bends have a curvature radius of the channel centerline $\left(R_{2}\right)$ of $2.25 \mathrm{~mm}$. Between two adjacent $180^{\circ}$ bends, there is a straight section of a length of $13.35 \mathrm{~mm}$ connecting them. As shown in Fig. 2c, the straight and serpentine microchannels have the same quasi-trapezoidal cross sectional dimensions, with a long base size, a short base size and a height of $1140 \pm 20 \mu \mathrm{m}, 990 \pm 20 \mu \mathrm{m}$, and $200 \pm 20 \mu \mathrm{m}$, respectively.

Two liquid-liquid systems were employed. Fluid properties are given in Table 2. The organic solvents are 1-butanol (Acros Organics, $\geq 99.5 \%$ ) and $n$-hexane (Acros Organics, $\geq$ $99.36 \%$ ), flowing into the cross-junction through the central microchannel. The aqueous phase is de-ionized water, which is fed from the two side microchannels. The soluble nature of 1-butanol in water is expected to present a negligible effect on flow patterns considering the short contact time between the two liquids, especially at the inlet junction. Minute amount of phenol red was added into water for a better clarity between the two phases. The flow patterns and evolutions were visualized at seven visualization windows (VWs), i.e., for the straight microchannel VW1 (@ cross-junction) and VW2 in the microchannel of $30 \mathrm{~mm}$ downstream from the junction; for the serpentine microchannel, VW3 at the inlet junction, VW4 and VW6 in the central zone of straight sections between two $180^{\circ}$ bends, and VW5 and VW7 at $180^{\circ}$ bends, as indicated in Fig. 2b. Experimental works were performed at $20.0 \pm 0.5^{\circ} \mathrm{C}$.
Firstly, water was supplied to saturate the microchannel for $10 \mathrm{~min}$. Secondly, $Q_{\mathrm{o}}$ was maintained constant while increasing $Q_{\mathrm{a}}$. The uncertainties for the volumetric flow rates $Q_{\mathrm{o}}$ and $Q_{\mathrm{a}}$ were $\pm 0.5 \%$ according to the specified accuracy of the syringe pumps. Snapshots were captured at steady-state conditions. The test ranges of some dimensionless numbers are provided in Table 3. The microchannels were cleaned and dried properly when completing each set of measurements.

\section{Results and discussion}

\subsection{Flow patterns at the inlet cross-junction}

Tubing/threading, dripping and jetting were identified at inlet junctions VW1 and VW3, as seen in Fig. 3. For a detailed description of their flow characteristics, see Wu et al. [11].

Figure $3 \mathrm{~b}$ and e show typical flow configurations of the dripping regime. The imposed flow of water from the two side microchannels at an angle of $45^{\circ}$ exerts a force on the organic phase with one vertical force component and one horizontal force component. As $Q_{\mathrm{a}}$ increases at a relatively low $Q_{\mathrm{o}}$, on one hand, the vertical force component due to imposed feeding flow of water increases and thus narrows and squeezes the organic phase when the front of the organic core thread partially obstructs the main microchannel. Therefore, a curved neck region is formed which connects the organic feeding flow and the organic core front. On the other hand, the horizontal force component tends to promote stretching of the organic core as the main microchannel is mostly obstructed by the front of the organic core. Besides, the organic core

Table 3 Operating dimensionless numbers for straight and serpentine microchannels

\begin{tabular}{|c|c|c|c|c|c|c|c|c|}
\hline Liquid-liquid system & $q$ & $\operatorname{Re}_{\mathrm{a}}, \rho_{\mathrm{a}} j_{\mathrm{a}} d_{\mathrm{h}} / \mu_{\mathrm{a}}$ & $\operatorname{Re}_{\mathrm{o}}, \rho_{\mathrm{o}} j_{\mathrm{o}} d_{\mathrm{h}} / \mu_{\mathrm{o}}$ & $\mathrm{Ca}_{\mathrm{a}}, \mu_{\mathrm{a}} \mathrm{j}_{\mathrm{a}} / \gamma$ & $\mathrm{Ca}_{\mathrm{o}}, \mu_{\mathrm{o}} \mathrm{j}_{\mathrm{o}} / \gamma$ & $\mathrm{We}_{\mathrm{a}}, \rho_{\mathrm{a}} j_{\mathrm{a}}^{2} d_{\mathrm{h}} / \gamma$ & $\mathrm{We}_{\mathrm{o}}, \rho_{\mathrm{o}} j_{\mathrm{o}}^{2} d_{\mathrm{h}} / \gamma$ & $\mathrm{Ce}_{\mathrm{o}}, \rho_{\mathrm{o}} u^{2} d_{\mathrm{h}}^{2} /(\gamma R)$ \\
\hline \multicolumn{9}{|l|}{ Straight microchannel } \\
\hline water-butanol & $1.0 \mathrm{E}-2 \sim 12$ & $0.22 \sim 130$ & $0.06 \sim 1.44$ & $3.6-4 \sim 0.22$ & $1.1 \mathrm{E}-3 \sim 2.6 \mathrm{E}-2$ & $7.9 \mathrm{E}-5 \sim 28.3$ & $6.4 \mathrm{E}-5 \sim 3.7 \mathrm{E}-2$ & - \\
\hline water-hexane & $6 \mathrm{E}-3 \sim 12$ & $0.22 \sim 1.30$ & $0.48 \sim 11.4$ & 1.3E-5 7.7E-3 & $3.8 \mathrm{E}-6 \sim 9.2 \mathrm{E}-5$ & $2.8 \mathrm{E}-6 \sim 1.0$ & $1.8 \mathrm{E}-6 \sim 1.0 \mathrm{E}-3$ & - \\
\hline \multicolumn{9}{|c|}{ Serpentine microchannel } \\
\hline water-butanol & $1.0 \mathrm{E}-2 \sim 96$ & $0.22 \sim 130$ & $0.06 \sim 5.76$ & $3.0 \mathrm{E}-5 \sim 6.0 \mathrm{E}-2$ & $5.6 \mathrm{E}-4 \sim 5.6 \mathrm{E}-2$ & 7.9E-5 28.4 & $6.4 \mathrm{E}-5 \sim 0.59$ & $8.5 \mathrm{E}-5 \sim 4.6$ \\
\hline water-hexane & $1.0 \mathrm{E}-2 \sim 24$ & $0.3 \sim 157$ & $0.7 \sim 68$ & $1.9 \mathrm{E}-5 \sim 9.2 \mathrm{E}-3$ & $5.8 \mathrm{E}-6 \sim 5.5 \mathrm{E}-4$ & $6.2 \mathrm{E}-6 \sim 1.44$ & 4.1E-6 3.8E-2 & $2.4 \mathrm{E}-6 \sim 0.15$ \\
\hline
\end{tabular}


Fig. 3 Flow pattern photos at cross-junctions, for the straight microchannel at VW1 a tubing, $Q_{\mathrm{o}}=15.0 \mathrm{ml} / \mathrm{h}$ for hexane and $Q_{\mathrm{a}}=4.0 \mathrm{ml} / \mathrm{h}$, b dripping, $Q_{\mathrm{o}}=$ $3.0 \mathrm{ml} / \mathrm{h}$ for hexane and $Q_{\mathrm{a}}=$ $45.0 \mathrm{ml} / \mathrm{h}$, and $\mathbf{c}$ jetting, $Q_{\mathrm{o}}=$ $1.0 \mathrm{ml} / \mathrm{h}$ for butanol and $Q_{\mathrm{a}}=$ $60.0 \mathrm{ml} / \mathrm{h}$; and for the serpentine microchannel at VW3 d tubing, $Q_{\mathrm{o}}=3.0 \mathrm{ml} / \mathrm{h}$ for butanol and $Q_{\mathrm{a}}=6.0 \mathrm{ml} / \mathrm{h}$, e dripping, $Q_{\mathrm{o}}=$ $3.0 \mathrm{ml} / \mathrm{h}$ for hexane and $Q_{\mathrm{a}}=$ $20.0 \mathrm{ml} / \mathrm{h}$, and $\mathbf{f}$ jetting, $Q_{\mathrm{o}}=$ $3.0 \mathrm{ml} / \mathrm{h}$ for butanol and $Q_{\mathrm{a}}=$ $80.0 \mathrm{ml} / \mathrm{h}$

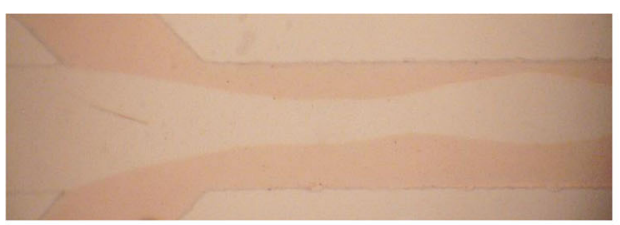

(a)

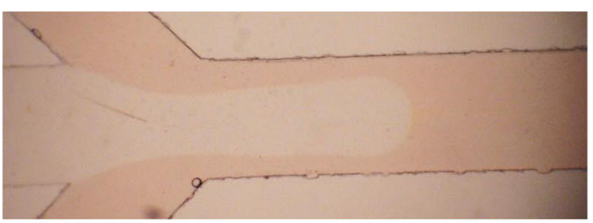

(b)

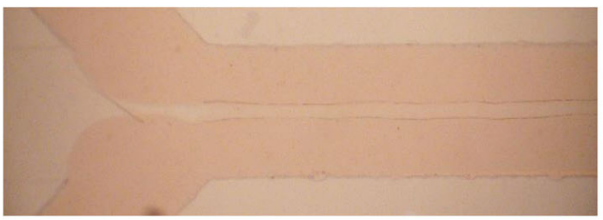

(c)

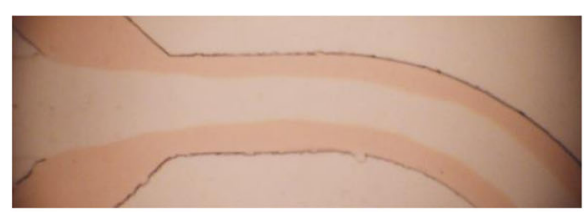

(d)

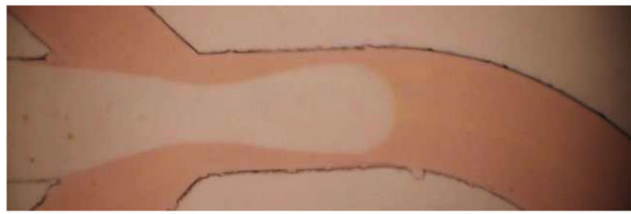

(e)

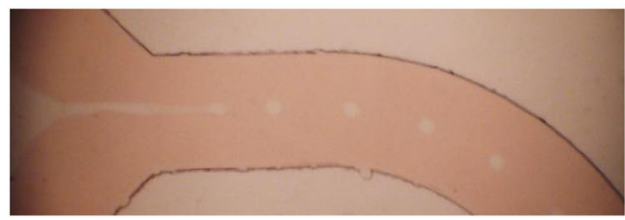

(d) stretches further into the main microchannel due to the viscous shear force generated by the water film flow between the organic phase front and the microchannel walls. Therefore, the neck becomes thinner and the radii of curvature at the neck region decrease. Thus, the interfacial tension force increases and dominates to destabilize the interface at the neck region. Therefore, the neck breaks up into slugs or droplets and these detached organic components are convected downstream.

If $Q_{\mathrm{o}}$ is comparably larger than $Q_{\mathrm{a}}$, tubing/threading regime prevails. As shown in Fig. 3a and d, there are a continuous organic core flow and an annulus aqueous film flow. As seen from Fig. 3a, small undulations fluctuate along the interface with time and space at a relatively high flow rate of hexane. However, no thread breakup occurs within the viewing zone of $5 \mathrm{~mm} \times$ $5 \mathrm{~mm}$. At high $Q_{\mathrm{o}}$, it is hard to form an organic neck region in the cross-junction, which is typical of the dripping regime, as the liquid inertia of the organic phase at a high flow rate dominates over the vertical force component generated by the imposed continuous flow of water from the side microchannels at a comparably low flow rate. In other words, the interfacial tension force diminishes in tubing/threading compared to dripping. Besides, as the organic core flows faster than the annulus water flow, there is a viscous shear force exerted on the organic core, trying to decelerate and hold back the organic core flow. The viscous shear force is approximately balanced by the horizontal force component because of feeding of the aqueous phase which tries to push the organic core downwards.

When $Q_{\mathrm{a}}$ increases further at a relatively low $Q_{\mathrm{o}}$, jetting occurs (Fig. 3c and f). During jetting, the organic phase thread can penetrate into the main microchannel as a single jet, whose length is normally larger than the junction size and up to several times of the channel size before droplet pinchoff at the tip [14]. The jet diameter is typically much less than the channel size. In jetting, the vertical force component generated by the fast imposed aqueous flow quickly thins the organic core thread in the junction, while the horizontal force component quickly carries the thin organic core thread downwards out of the inlet junction. The viscous shear from the continuous water flow further elongates the inner organic core thread, and interfacial instabilities are also convected downstream. Thus it is not able to develop a curved neck region at/ near the cross-junction similar to the one in dripping due to dominant aqueous inertia and its induced viscous shear over interfacial tension and organic inertia. Therefore, jetting prevails at a comparably high $Q_{\mathrm{a}}$ and a relatively low $Q_{\mathrm{o}}$. Further downstream in the microchannel, the jet propagates thread undulations induced by Rayleigh-Plateau instabilities and overcomes the viscous shear or inertia to generate slugs or droplets.

Phase superficial velocities or dimensionless numbers are generally used to map flow patterns. The $\mathrm{Ca}_{\mathrm{a}}\left(\mu_{\mathrm{a}} j_{\mathrm{a}} / \gamma\right)$ and $\mathrm{We}_{\mathrm{o}}$ 
Fig. 4 Flow pattern maps at the cross-junction VW1 based on $\mathrm{Ca}_{\mathrm{a}}$ and $\mathrm{We}_{\mathrm{o}}$

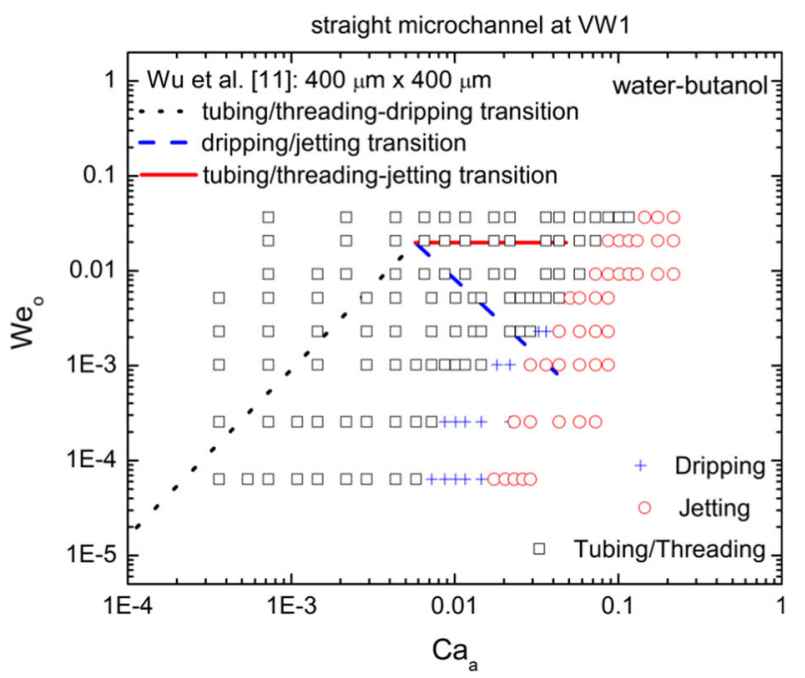

(a) water-butanol flow. The solid and dashed lines are flow pattern transitional lines extracted from Wu et al. [11].

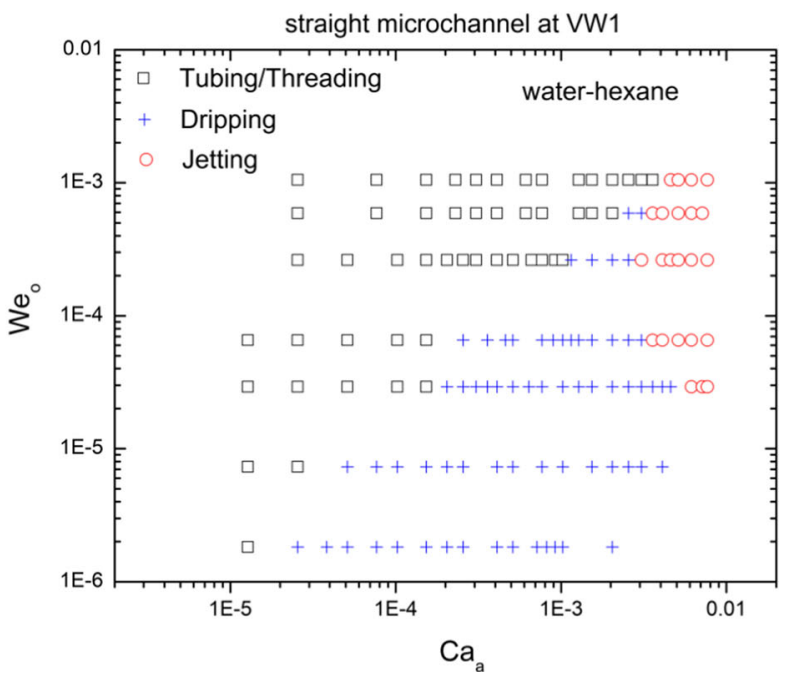

(b) water-hexane flow

$\left(\rho_{\mathrm{o}} j_{\mathrm{o}}^{2} d_{\mathrm{h}} / \gamma\right)$ were adopted to map the observed flow patterns at cross-junctions, where $j_{\mathrm{a}}$ and $j_{\mathrm{o}}$ are superficial velocities of aqueous and organic phases, respectively. The $d_{\mathrm{h}}$ is $335 \mu \mathrm{m}$, the same for the two microchannels, which was calculated as four times the cross-section area over the perimeter of the cross section. Figure 4 shows flow pattern maps at VW1 for the straight microchannel while Fig. 5 provides maps at VW3 for the serpentine microchannel.

For water-butanol flow in the straight microchannel as given in Fig. 4a, the flow regime is tubing or threading at low to medium $\mathrm{Ca}_{\mathrm{a}}$. At high $\mathrm{Ca}_{\mathrm{a}}$, the flow regime is jetting. There is a small dripping regime between the two flow regimes at low to medium $\mathrm{We}_{\mathrm{o}}$. At high $\mathrm{We}_{\mathrm{o}}$ or at high $\mathrm{Ca}_{\mathrm{a}}$, the dripping regime can hardly survive when either the organic liquid inertia or the aqueous viscous shear dominates over the interfacial tension force. The transitional lines from Wu et al. [11] for the same liquid-liquid system in square channels with a $d_{\mathrm{h}}$ of $400 \mu \mathrm{m}$ were shown in Fig. 4a for comparison. There are two major differences. The tubing/threading regime is much larger while the dripping regime is much narrower compared to those in $\mathrm{Wu}$ et al. [11]. Similarly for water-hexane flow, dripping prevails over the entire experimental range in Wu et al. [11] with a similar range of phase flow rates, while in this work dripping becomes much narrower (Fig. 4b). Tubing/threading dominates at low to medium $\mathrm{Ca}_{\mathrm{a}}$ and medium to high $\mathrm{We}_{\mathrm{o}}$. Jetting appears at high $\mathrm{Ca}_{\mathrm{a}}$. The smaller junction angle in the 


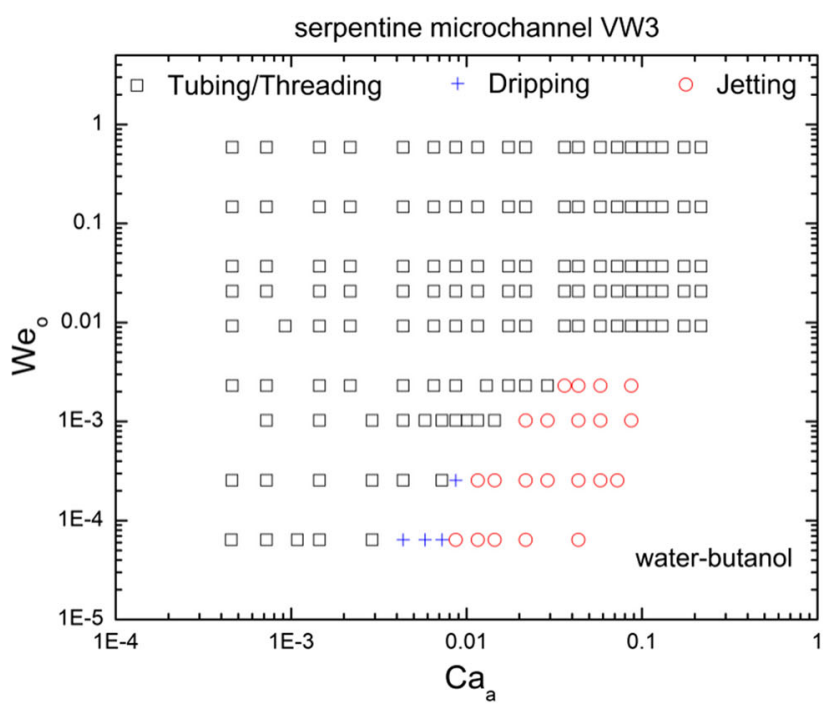

(a) water-butanol flow

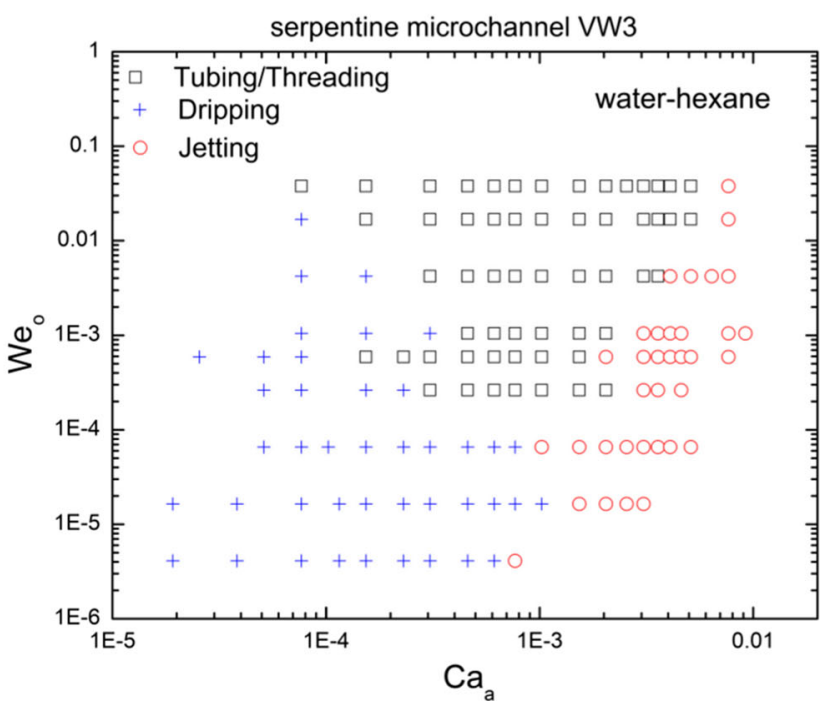

(b) water-hexane flow

Fig. 5 Flow pattern maps at the cross-junction VW3 based on $\mathrm{Ca}_{\mathrm{a}}$ and $\mathrm{We}_{\mathrm{o}}$

present work probably attributes to the expansion of tubing/ threading and the narrowing of dripping. In Wu et al. [11], the three feeding microchannels intersect at right angles, i.e., with a junction angle of $90^{\circ}$, while in the present work the two side microchannels meet the central microchannel with the same junction angle of $45^{\circ}$. Considering the two works with similar cross-sectional areas and the same water flow rate, in Wu et al. [11] the vertical force component is much larger, accelerating the thinning of the organic core thread in the junction, while the horizontal force component is much less, providing longer time for the front of the organic core thread to fill up the main microchannel. Therefore, it is much easier to form a curved thin neck in Wu et al. [11] than in this study, which indicates that dripping covers a much larger zone in Wu et al. [11] while instead tubing/threading covers a much larger region in this work. Comparing water-butanol and water-hexane flows, the former has a much smaller dripping regime mainly because the former has a much less interfacial tension.

At VW3 of the serpentine microchannel, the three regimes mentioned above were observed, as shown in Fig. 5. The first $90^{\circ}$ bend, $2.8 \mathrm{~mm}$ downstream from the cross-junction at VW3, redistributes the phases and gradually changes the flow direction by $90^{\circ}$, which might promote an asymmetric upstream flow to the channel centerline at the cross-section at low flow rates of the aqueous phase. By comparing Figs. $4 \mathrm{~b}$ and $5 \mathrm{~b}$ for water-hexane flow, the tube/threading regime becomes narrower while the dripping regime becomes larger especially at low $\mathrm{We}_{\mathrm{o}}$ values. The centrifugal force changes the flow direction, produces chaotic advection and improves phase interaction to enhance break-up of the organic core thread at the $90^{\circ}$ bend, thus causing transition from tubing/ threading to dripping. As shown in Fig. 4a for the straight microchannel and in Fig. 5a for the serpentine microchannel, water-butanol flow is much less affected by the downstream bend compared to water-hexane flow. The interfacial tension of water-hexane flow is one order of magnitude larger than that of water-butanol flow. Accordingly, interfacial instability is much more intense and apparent for the former than the latter. The asymmetrical flow at the cross-junction and the directional change of the flow are supposed to augment interfacial disturbances and waves in water-hexane flow.

\subsection{Flow patterns in the main microchannel}

Flow patterns were observed at VW2 for the straight microchannel, and at VW4, VW5, VW6 and VW7 for the serpentine microchannel. Three main flow patterns, i.e., annular, slug and droplet flows were observed for the two channels. For example, Fig. 6 shows several typical flow patterns observed at VW5 of the serpentine microchannel. Flow patterns for the straight microchannel at VW2 and for the serpentine microchannel at VW6 were mapped by using the organic phase and aqueous phase superficial velocities, as shown in Figs. 7 and 8, respectively. In annular flow, the organic phase flows continuously in the core of the microchannel, surrounded by the annulus aqueous phase, e.g., Fig. 6a. As the water flow rate increases, the organic core thread becomes thinner. The thin annular core can be stable up to a high water flow rate, e.g., Fig. 6b. If the organic core thread breaks up into slugs in the junction or close to the inlet or even after a certain distance depending on flow conditions, slug flow was observed. Interfacial tension dominates slug flow. The slug 
shape depends on $\mathrm{Ca}_{\mathrm{a}}$ and flow rate ratios. The slug length is normally larger than the hydraulic diameter of the microchannel, see., e.g., Fig. 6c. Periodic deformations of the slug end were noticed in the serpentine microchannel due to centrifugal forces, which was also observed in Roudet et al. [24] for a meandering microchannel. In Fig. 6d, at the same $Q_{\mathrm{o}}$ of hexane, a larger $Q_{\mathrm{a}}$ exerts a larger pressure and a larger viscous shear on the interface, accelerating the neck thinning and break-up to form slugs and droplets. Smaller droplets may coalesce to form larger droplets or slugs, which tend to attach channel walls. This phenomenon stems from the partial wetting behaviours of water and organic liquids on glass microchannel walls. Regular and ordered slug flow and droplet flow of uniform slug or droplet size may be achieved by adding surfactants to prevent the coalescence of droplets [29], which will be investigated in the near future. The slug-droplet flow shown in Fig. 6d was also categorized as slug flow in the present study. As $Q_{\mathrm{a}}$ further increases, the slug size decreases and droplets are formed depending on the liquid-liquid systems. Figure 6e shows droplet flow for waterbutanol in the serpentine microchannel. From visualization, the organic jet was observed to break up near the inlet junction and droplet flow was formed.

For the straight microchannel, the annular flow at VW2 corresponds to most of the tubing/threading regime observed at VW1; the slug flow at VW2 occupies a large fraction of the dripping regime and a small fraction of the tubing/threading regime at VW1; and the droplet flow at VW2 covers the
Fig. 6 Flow pattern photos at VW5 a annular flow, $Q_{\mathrm{o}}=3.0 \mathrm{ml} /$ $\mathrm{h}$ for butanol and $Q_{\mathrm{a}}=6.0 \mathrm{ml} / \mathrm{h}, \mathbf{b}$ annular flow, $Q_{\mathrm{o}}=3.0 \mathrm{ml} / \mathrm{h}$ for butanol and $Q_{\mathrm{a}}=40.0 \mathrm{ml} / \mathrm{h}$, c slug flow, $Q_{\mathrm{o}}=3.0 \mathrm{ml} / \mathrm{h}$ for hexane and $Q_{\mathrm{a}}=6.0 \mathrm{ml} / \mathrm{h}$, d slug flow, $Q_{\mathrm{o}}=3.0 \mathrm{ml} / \mathrm{h}$ for hexane and $Q_{\mathrm{a}}=40.0 \mathrm{ml} / \mathrm{h}$, e droplet flow, $Q_{\mathrm{o}}=3.0 \mathrm{ml} / \mathrm{h}$ for butanol and $Q_{\mathrm{a}}=80.0 \mathrm{ml} / \mathrm{h}$

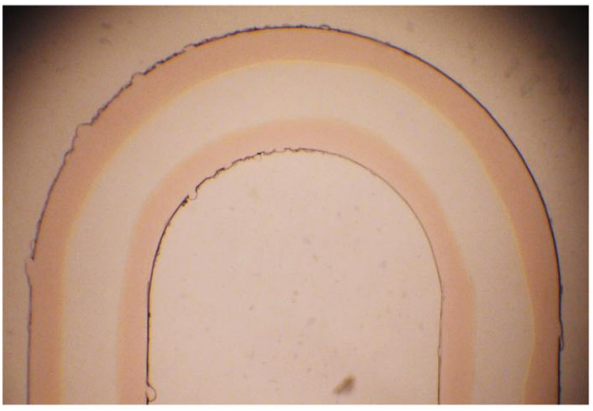

(a)

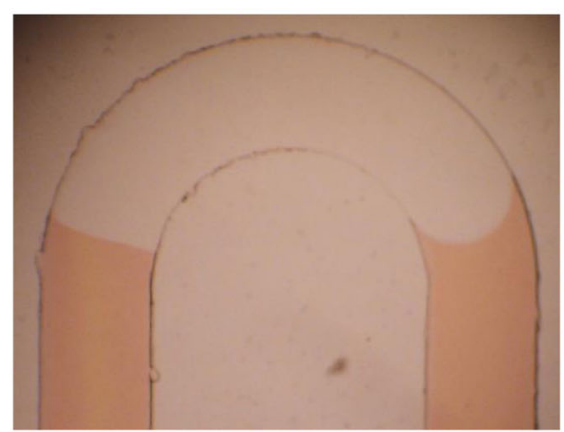

(c)

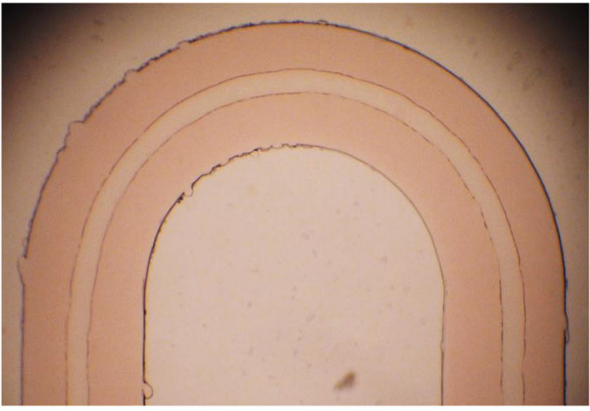

(b)

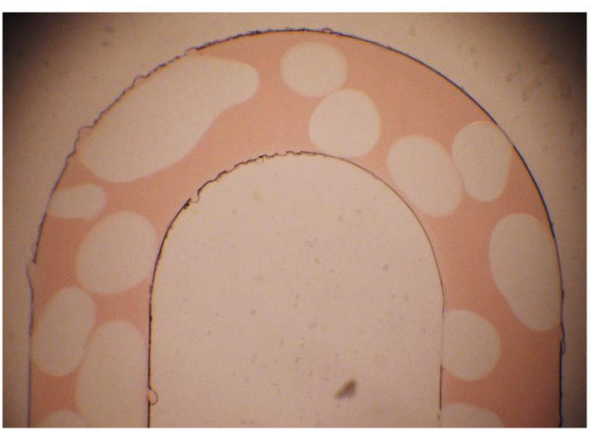

(d)

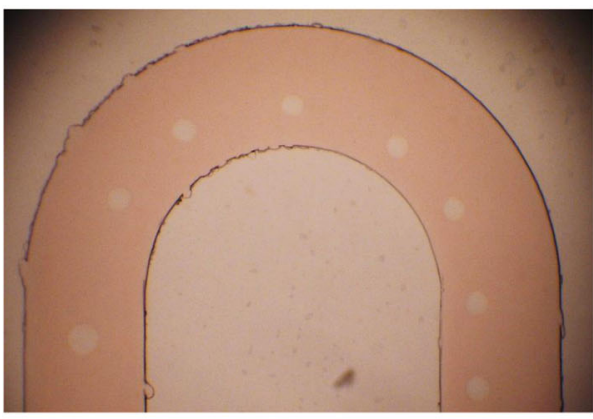

(e) 
jetting regime and a small fraction of the dripping regime at VW1. Therefore, the reasoning regarding flow pattern transitions at the inlet junction presented above is still valid for flow pattern transitions downstream in the main microchannel.

Comparing Fig. 7 at VW2 and Fig. 8 at VW6, the slug flow regime at VW6 is larger than that at VW2. The change of the flow direction in the serpentine microchannel causes a centrifugal force, generating chaotic advection and enhancing phase interaction at and near the bends. Besides, there is a tendency to force the heavier fluid towards the outside and the lighter fluid towards the inner side of the bend, though this movement is not noticeable from flow pattern images for most cases as

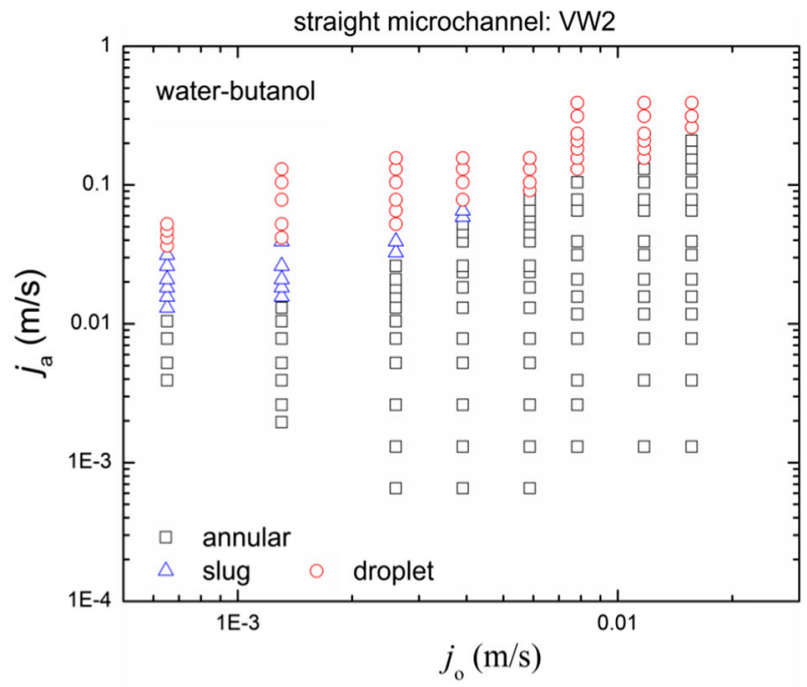

(a) water-butanol flow

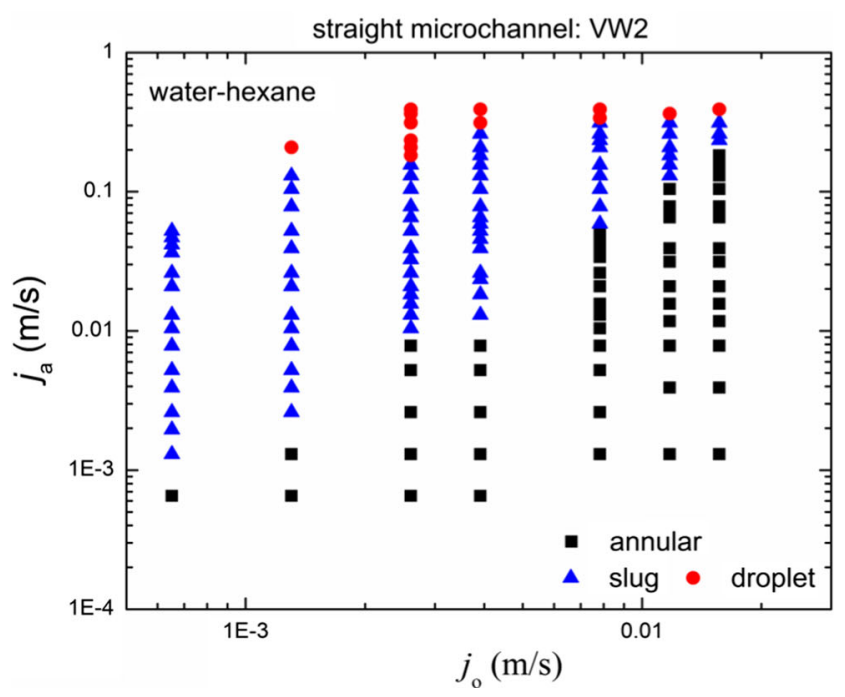

(b) water-hexane flow

Fig. 7 Flow pattern maps at VW2 based on $j_{\mathrm{o}}$ and $j_{\mathrm{a}}$

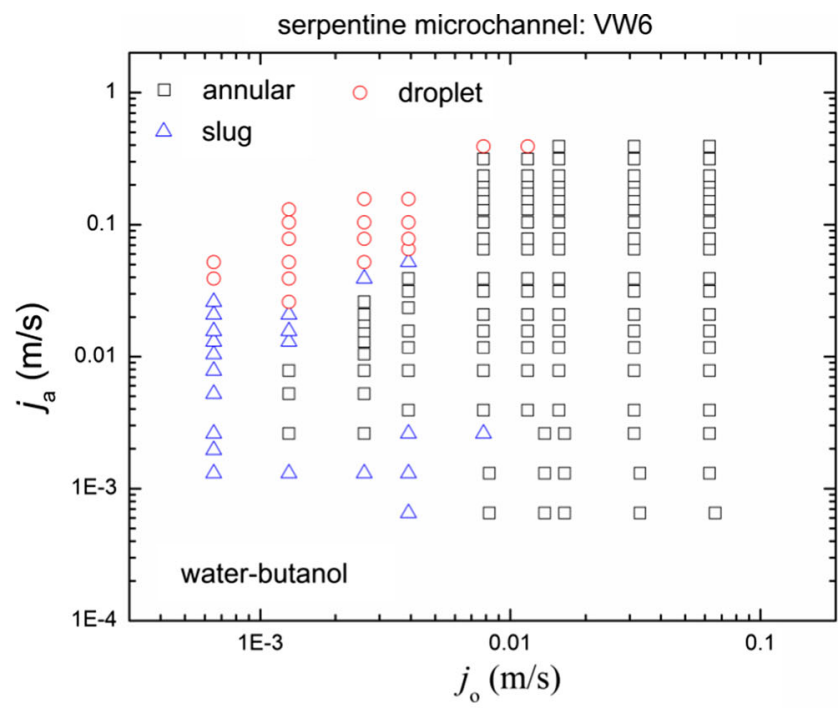

(a) water-butanol flow

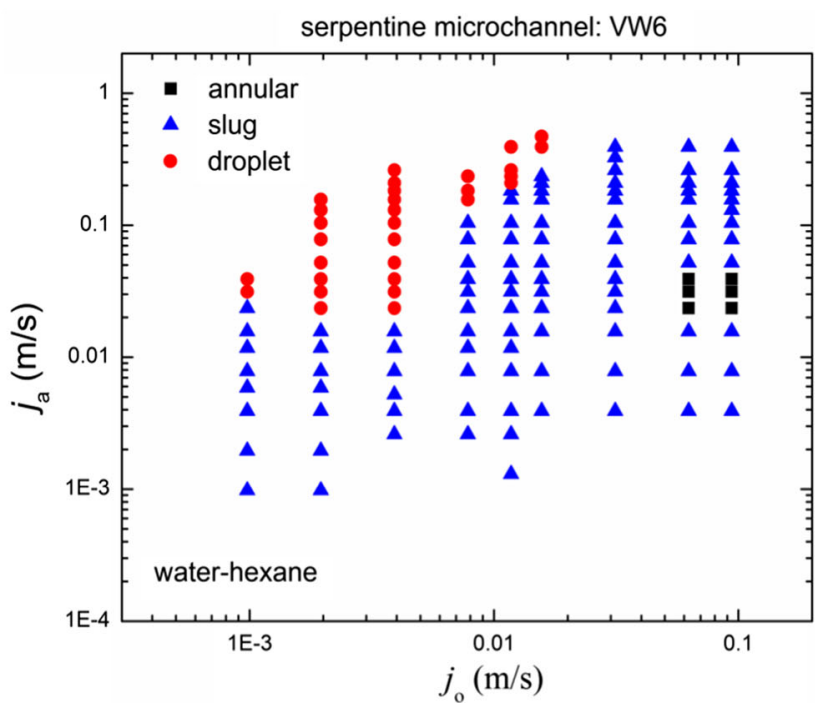

(b) water-hexane flow

Fig. 8 Flow pattern maps at VW6 based on $j_{\mathrm{o}}$ and $j_{\mathrm{a}}$

the density values of the organic and aqueous phases do not differ much. Such liquid movement tends to give rise to a slip between the phases and complicates the flow structure. As a result, depending on flow conditions, the organic annular core breaks up into slugs and long slugs break up into short slugs and droplets. Therefore, flow pattern may vary along the flow direction due to centrifugal forces in bend regions. For example, at $Q_{\mathrm{o}}=6 \mathrm{ml} / \mathrm{h}$ and $Q_{\mathrm{a}}=30 \mathrm{ml} / \mathrm{h}$, annular flow occurred and the organic core is continuous over VW4, while slug flow was observed at VW6 after flowing through four $180^{\circ}$ bends. Therefore, slug flow prevails over a larger regime while annular flow shrinks at VW6 in the serpentine microchannel. 
Fig. 9 Ranges of several dimensionless numbers, showing the ratios of the buoyancy force to the interfacial tension $(\mathrm{Bo})$, the inertia force to the interfacial tension (We), the viscous shear force to the interfacial tension (Ca), and the centrifugal force to the interfacial tension $(\mathrm{Ce})$

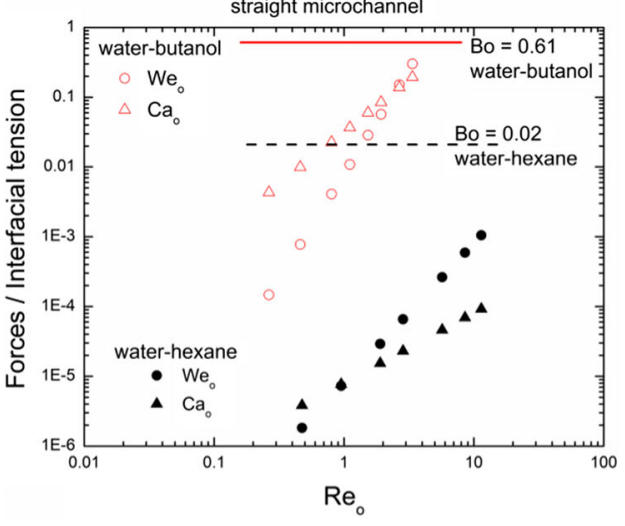

(a)

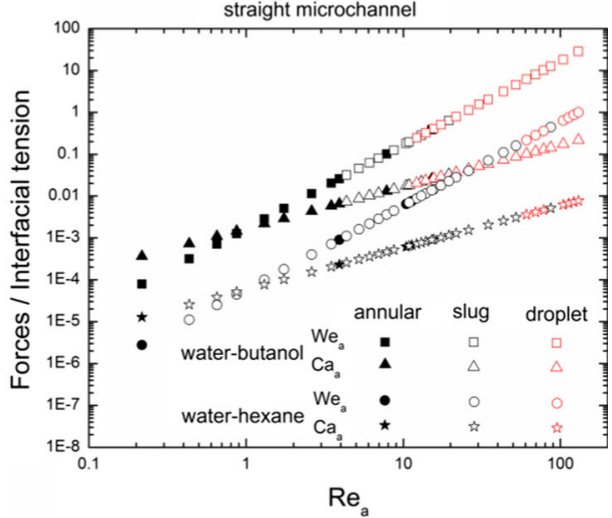

(b)

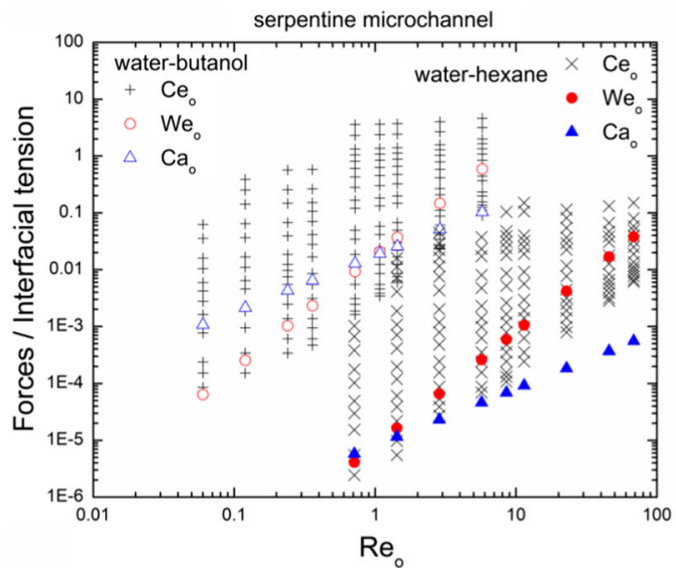

(c)

Dimensionless numbers are also employed to map flow patterns in microchannels. To begin with, Fig. 9 presents the operating ranges of dimensionless numbers, i.e., $\mathrm{We}, \mathrm{Ca}, \mathrm{Bo}$ and $\mathrm{Ce}$. The $\mathrm{Ce}$ number indicates a comparison between centrifugal forces and interfacial tension forces. The $u$ was used in the Ce number instead of superficial velocities. Figure $9 \mathrm{a}$ and $\mathrm{b}$ gives comparisons of dimensionless numbers for the two phases flowing in the straight microchannel. The $\mathrm{We}_{\mathrm{o}}$ and $\mathrm{Ca}_{\mathrm{o}}$ numbers of water-butanol flow are at least two orders of magnitude larger than those of water-hexane flow, at a similar range of $\operatorname{Re}_{\mathrm{o}}$ from 0.1 to 10 , suggesting that interfacial tension is significant for water-hexane flow even at a high $\mathrm{Re}_{\mathrm{o}}$. For the aqueous phase, the relatively higher $\mathrm{We}_{\mathrm{a}}$ values at high $\mathrm{Re}_{\mathrm{a}}$ indicate that the liquid inertia of the aqueous phase tends to present a considerable effect on the organic phase. As for the serpentine microchannel in Fig. 9c for the organic phase, the ranges of $\mathrm{We}_{\mathrm{o}}$ and $\mathrm{Ca}_{\mathrm{o}}$ are similar to those for the straight microchannel. For each $\mathrm{Re}_{\mathrm{o}}$, Ce increases when the average flow velocity increases. It seems that effect of the centrifugal forces at and near the bends are important, especially at

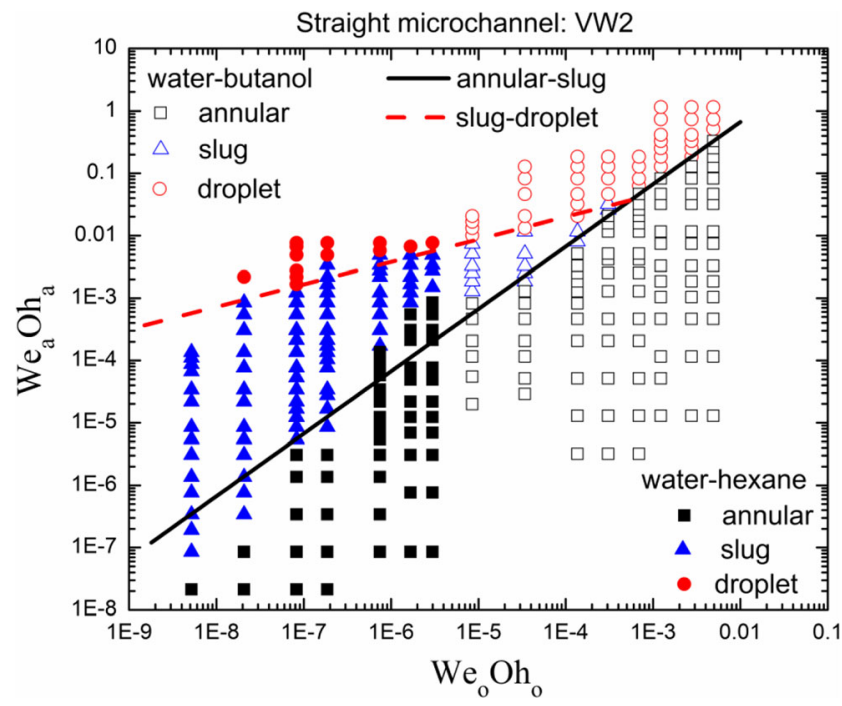

Fig. 10 Flow pattern maps at VW2 based on a combined dimensionless number WeOh. The solid and dashed lines correspond to the new flow pattern transitional lines based on the experimental data 
Fig. 11 Comparisons of the newly developed flow pattern transitional lines (the solid and dashed lines) for the straight microchannel at VW2 to those flow pattern transitional data points in Cao et al. [30] for waterbutanol and water-oil flows in a square microchannel

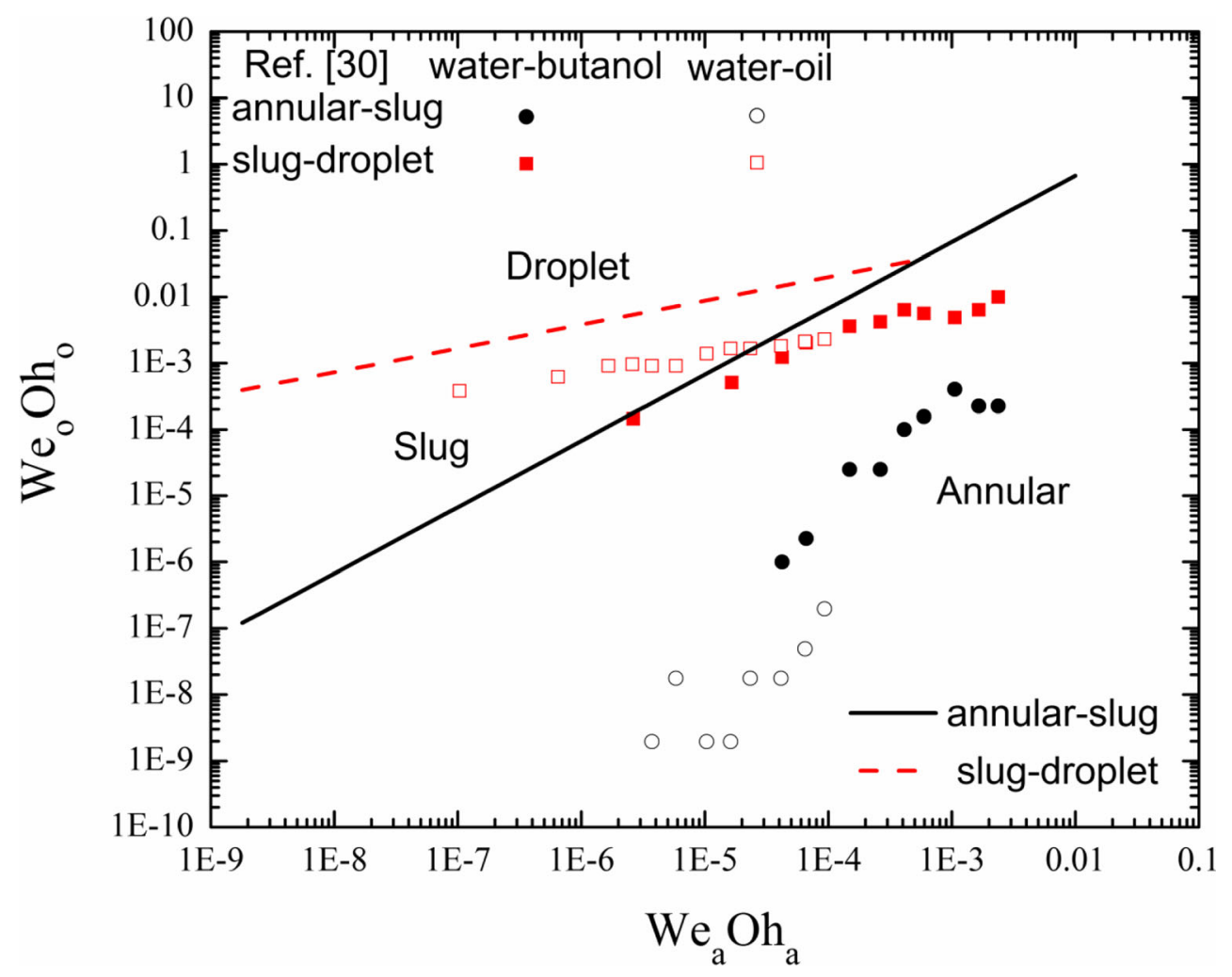

relatively high flow velocities. For the serpentine microchannel, the $\mathrm{We}_{\mathrm{a}}$ and $\mathrm{Ca}_{\mathrm{a}}$ ranges of the aqueous phase are similar to those in Fig. $9 \mathrm{~b}$ and the Ce range is similar to that in Fig. 9c. Therefore, comparison of non-dimensional numbers for the aqueous phase is not given here.

The dimensionless group $\mathrm{WeOh}$, proposed by Yagodnitsyna et al. [29] for water-ionic liquid flow in microchannels, was used to present flow pattern observations. The Ohnesorge number $(\mathrm{Oh})$ equals $\mathrm{Ca}$ over the square root of We. Figure 10 shows water-butanol and water-hexane flows at VW2 of the straight microchannel. Flow pattern transitional lines can be obtained from the flow pattern map for the two liquid-liquid systems. The red dashed line indicates the slug-droplet transition, while the black solid line indicates the annular-slug transition and also the annular-droplet transition at high $\mathrm{We}_{\mathrm{a}} \mathrm{Oh}_{\mathrm{a}}$ values. It can be seen from Fig. 10 that the newly developed transitional lines can estimate the flow pattern data of both water-butanol flow and water-hexane flow. The transitional lines were compared with the flow pattern transition points in Cao et al. [30] for water-butanol and water-oil flows in a square cross-junction channel with a junction angle of $90^{\circ}$ and a channel width of $400 \mu \mathrm{m}$, as shown in Fig. 11. In general, the annular flow is much larger and the slug flow is much smaller at VW2 of the straight microchannel than those of the square microchannel in Cao et al. [30]. The difference at the inlet junction, i.e., the junction angle of the quasi-trapezoidal straight microchannel in the present work and the square microchannel in Cao et al. [30] is one of the main reasons attributing to this phenomenon, with a similar argument as for Fig. 4a.

Flow pattern maps employing dimensionless number groups $\mathrm{We}_{\mathrm{o}} \mathrm{Oh}_{\mathrm{o}}$ and $\mathrm{We}_{\mathrm{a}} \mathrm{Oh}_{\mathrm{a}}$ were shown in Fig. 12 for VW4-VW7 of the serpentine microchannel. The transitional lines for the straight microchannel at VW2 were shown for comparison in Fig. 12a for VW4, a straight section after a $90^{\circ}$ bend and an $180^{\circ}$ bend. The three flow patterns are located in zones similar to those in the straight microchannel. However, the slug flow regime becomes larger towards the annular flow regime and the droplet flow regime becomes slightly larger towards the slug flow regime at VW4 because the centrifugal force might break up the organic annular core into slugs and also break up the slugs into droplets over the bends. Based on the same reasoning, by comparing the flow pattern maps along the flow direction of the serpentine microchannel from VW4 to VW7, the slug flow regime expands further towards the annular flow regime after more $180^{\circ}$ bends, while the droplet flow regime expands slightly.

Effect of the aspect ratio on flow patterns is emphasized as follows. It is supposed that with similar cross-sectional areas and hydraulic diameters, similar inlet junctions and the same phase flow rates, a rectangular microchannel might promote 


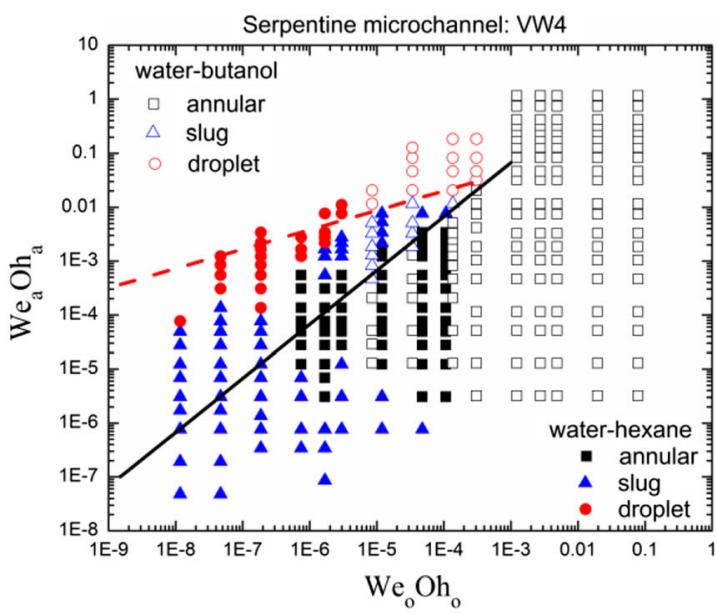

(a)

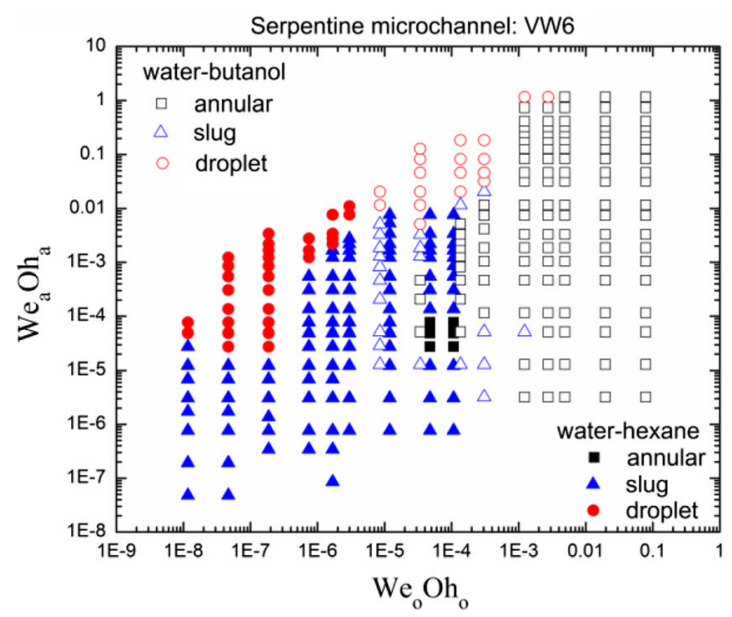

(c)

Fig. 12 Flow pattern maps for the serpentine channel based on a combined dimensionless number $\mathrm{WeOh}$ along the flow direction at: a VW4, b VW5, c VW6, and d VW7. The solid and dashed lines in Fig.

tubing/threading near the junction and annular flow in the downstream microchannel. Figure 13 shows conceptual cross-sectional film distributions for the quasi-trapezoidal microchannel with an aspect ratio of 5.3, considering that the short and long bases do not differ much. At a relatively high Capillary number of the aqueous phase, above a transitional value of 0.01 , Fig. 13a shows that the cross-sectional profile of the organic core looks like an ellipse or an oval as the viscous shear overcomes the interfacial tension force. At a relatively low capillary number, i.e., less than 0.01 , depending on the aqueous inertia force, the cross-sectional profile of the organic phase might look like either the one shown in Fig. 13b at a relatively low $Q_{\mathrm{a}}$ or the one shown in Fig. 13c at a relatively high $Q_{\mathrm{a}}$. In Fig. 13b, the film thickness is still relatively thick in the plane of the longer semi-axis. When the inertia of the

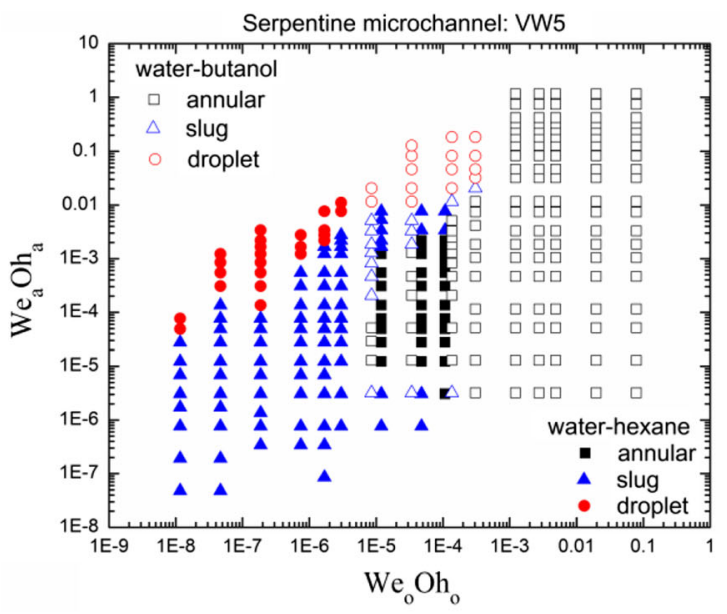

(b)

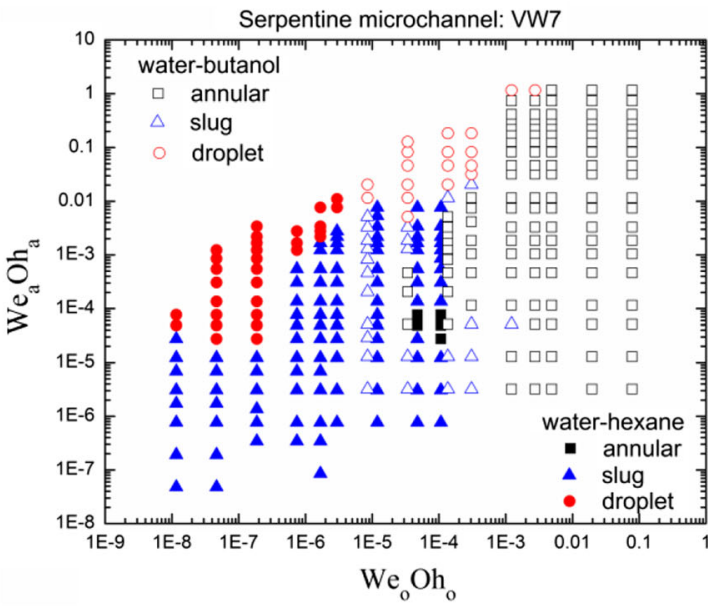

(d)

12a correspond to the newly developed annular-slug and the slug-droplet transitional lines for the straight microchannel at VW2, respectively

aqueous phase increases, the interface might become arcshaped in the plane of the longer semi-axis, with an even larger gap for film flow of the aqueous phase [31]. Such a large aqueous leakage flow around the organic core thread in rectangular and quasi-trapezoidal microchannels of high aspect ratios is very favorable for the persistence of tubing/threading in the cross-junction and annular flow in the downstream channel. Firstly, at the inlet junction, the force exerted by continuously imposed water flow on organic core thread decreases because a part of or most of the aqueous phase leaks through from the corners and gaps as shown in Fig. 13b and c. Besides, the resulting less aqueous flow between the front of the organic core thread and the microchannel walls gives a smaller velocity gradient normal to the wall and therefore a less shear force on the organic core front, which means a slower neck thinning and 


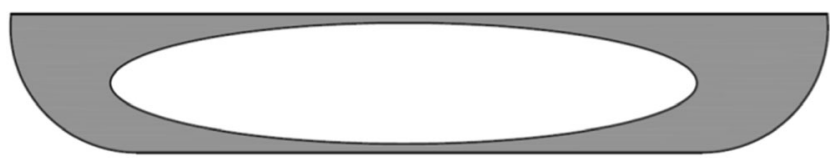

(a)

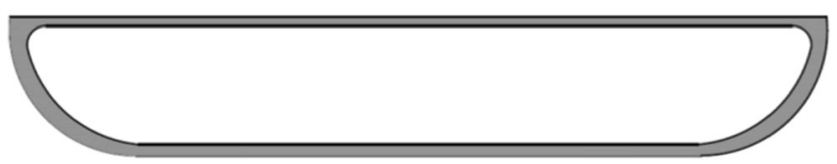

(b)

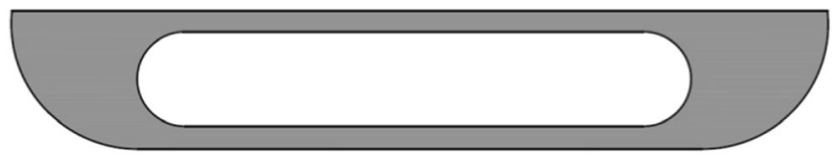

(c)

Fig. 13 Conceptual cross-sectional film distributions for quasitrapezoidal microchannels of high aspect ratios at a $\mathrm{Ca}$ (calculated as $\mu_{\mathrm{a}} \mathrm{u} / \gamma$ for this case): a much larger than $0.01, \mathbf{b}$ much less than 0.01 with a low or moderate $Q_{\mathrm{a}}$, and $\mathbf{c}$ less than 0.01 with a relatively high $Q_{\mathrm{a}}$

thus no break-up of the organic core. From another perspective, for slug flow in the main microchannel, due to leakage flow, the amount of liquid in aqueous slugs between two organic slugs would reduce and therefore coalesce of organic slugs is more likely to occur to form annular flow.

\section{Conclusions}

Water-butanol and water-hexane two-phase flows in ultrashallow straight and serpentine microchannels with a crossjunction were visualized for wide ranges of superficial velocities. The two microchannels have the same quasi-trapezoidal profile and the same $d_{\mathrm{h}}$ of $335 \mu \mathrm{m}$. The aspect ratio is as high as 5.3. At the inlet junction, three major flow patterns including tubing/threading, dripping and jetting were presented using $\mathrm{Ca}_{\mathrm{a}}$ versus $\mathrm{We}_{\mathrm{o}}$. Correspondingly, in the main microchannel, three main flow patterns were categorized, i.e., annular, slug and droplet flows. A dimensionless number group, Weber number times Ohnesorge number, was adopted to map flow regimes in the downstream microchannel. The flow pattern map, i.e., $\mathrm{We}_{\mathrm{a}} \mathrm{Oh}_{\mathrm{a}}$ versus $\mathrm{We}_{\mathrm{o}} \mathrm{Oh}_{\mathrm{o}}$, can roughly capture liquid-liquid flow patterns. Flow pattern transitional lines for water-butanol and water-hexane flows in the main microchannel were obtained.

The flow pattern transitions were explained based on a force analysis. For the studied cross-junction with a junction angle of $45^{\circ}$, the vertical force component of the inertia due to the imposed water flow narrows and squeezes the organic core thread while its horizontal force component and the viscous shear promote stretching of the organic core. These forces together with the interfacial tension force of the neck connecting the feeding flow and the front of the organic core thread, and the liquid inertia of the organic phase shape the curved neck region at the inlet junction and determine whether the organic core thread breaks up or not and the resulting flow regimes. Compared to a junction angle of $90^{\circ}$, a junction angle of $45^{\circ}$ tends to provide a larger tubing/threading regime at the inlet junction and a wider annular flow regime. Besides, a large aspect ratio is also favorable for tubing/threading at the inlet junction and downstream annular flow because of a large aqueous leakage flow around the organic core through the corners and gaps.

Compared to the straight microchannel, the slug flow regime becomes larger towards the annular flow regime and the droplet flow regime becomes slightly larger towards the slug flow regime in serpentine microchannels because the centrifugal force tends to break up the organic annular core into slugs and also break up the slugs into droplets over the bends.

Acknowledgments The authors appreciate financial support from the Swedish Research Council (VR).

\section{Compliance with ethical standards}

Conflict of interest On behalf of all authors, the corresponding author states that there is no conflict of interest.

Open Access This article is distributed under the terms of the Creative Commons Attribution 4.0 International License (http:// creativecommons.org/licenses/by/4.0/), which permits unrestricted use, distribution, and reproduction in any medium, provided you give appropriate credit to the original author(s) and the source, provide a link to the Creative Commons license, and indicate if changes were made.

Publisher's Note Springer Nature remains neutral with regard to jurisdictional claims in published maps and institutional affiliations.

\section{References}

1. Sattari-Najafabadi M, Nasr EM, Wu Z, Sundén B (2018) Mass transfer between phases in microchannels: A review. Chem Eng Process Process Intensif 127:213-237

2. Zhou M, Wang SF, Zhou Y (2017) Phase distribution of nitrogenwater two-phase flow in parallel micro channels. Heat Mass Transf 53(4):1175-1182

3. Mehta HB, Banerjee J (2014) An investigation of flow orientation on air-water two-phase flow in circular minichannel. Heat Mass Transf 50(10):1353-1364

4. Deng D, Wang RS, Xie SW, Liu PP (2015) Flow pattern and its transition of nitrogen gas-water two-phase flow in U-tubes and inverted U-tubes. Heat Mass Transf 51(1):85-94 
5. Saljoshi PS, Autee AT (2017) Experimental investigation of twophase flow patterns in minichannels at horizontal orientation. Heat Mass Transf 53(9):2799-2811

6. Zheng D, Che D (2007) An investigation on near wall transport characteristics in an adiabatic upward gas-liquid two-phase slug flow. Heat Mass Transf 43(10):1019-1036

7. Foroughi H, Kawaji M (2011) Viscous oil-water flows in a microchannel initially saturated with oil: Flow patterns and pressure drop characteristics. Int J Multiphase Flow 37:1147-1155

8. Sattari-Najafabadi M, Nasr EM, Wu Z, Sundén B (2017) Hydrodynamics and mass transfer in liquid-liquid non-circular microchannels: Comparison of two aspect ratios and three junction structures. Chem Eng J 322:328-338

9. Cao Z, Wu Z, Sundén B (2018) Dimensionless analysis on liquidliquid flow patterns and scaling law on slug hydrodynamics in cross-junction microchannels. Chem Eng J 344:604-615

10. Plouffe P, Roberge DM, Macchi A (2016) Liquid-liquid flow regimes and mass transfer in various micro-reactors. Chem Eng J 300: 9-19

11. Wu Z, Cao Z, Sundén B (2017) Liquid-liquid flow patterns and slug hydrodynamics in square microchannels of cross-shaped junctions. Chem Eng Sci 174:56-66

12. Kovalchuk NM, Roumpea E, Nowak E, Chinaud M, Angeli P, Simmons MJ (2018) Effect of surfactant on emulsification in microchannels. Chem Eng Sci 176:139-152

13. Nie Z, Seo M, Xu S, Lewis PC, Mok M, Kumacheva E, Whitesides GM, Garstecki P, Stone HA (2008) Emulsification in a microfluidic flow-focusing device: effect of the viscosities of the liquids. Microfluid Nanofluid 5:585-594

14. Nunes JK, Tsai SSH, Wan J, Stone HA (2013) Dripping and jetting in microfluidic multiphase flows applied to particle and fibre synthesis. J Phys D Appl Phys 46:114002

15. Cubaud T, Mason TG (2008) Capillary threads and viscous droplets in square microchannels. Phys Fluids 20:053302

16. De Menech M, Garstecki P, Jousse F, Stone HA (2008) Transition from squeezing to dripping in a microfluidic T-shaped junction. J Fluid Mech 595:141-161

17. Fu T, Wu Y, Ma Y, Li HZ (2012) Droplet formation and breakup dynamics in microfluidic flow-focusing devices: From dripping to jetting. Chem Eng Sci 84:207-217

18. Garstecki P, Fuerstman MJ, Stone HA, Whitesides GM (2006) Formation of droplets and bubbles in a microfluidic T-junction scaling and mechanism of break-up. Lab Chip 6:437-446
19. Kawaji M, Mori K, Bolintineanu D (2009) The effects of inlet geometry and gas-liquid mixing on two-phase flow in microchannels. ASME J Fluid Eng 131:041302

20. Günther A, Khan SA, Thalmann M, Trachsel F, Jensen KF (2004) Transport and reaction in microscale segmented gas-liquid flow. Lab Chip 4:278-286

21. Kirpalani DM, Patel T, Mehrani P, Macchi A (2008) Experimental analysis of the unit cell approach for twophase flow dynamics in curved flow channels. Int $\mathrm{J}$ Heat Mass Transf 51:1095-1103

22. Fries DM, von Rohr PR (2009) Liquid mixing in gas-liquid twophase flow by meandering microchannels. Chem Eng Sci 64:13261335

23. Dessimoz AL, Raspail P, Berguerand C, Kiwi-Minsker L (2010) Quantitative criteria to define flow patterns in micro-capillaries. Chem Eng J 160:882-890

24. Roudet M, Loubiere K, Gourdon C, Cabassud M (2011) Hydrodynamic and mass transfer in inertial gas-liquid flow regimes through straight and meandering millimetric square channels. Chem Eng Sci 66:2974-2990

25. Sarkar PS, Singh KK, Shenoy KT, Sinha A, Rao H, Ghosh SK (2012) Liquid-liquid two-phase flow patterns in a serpentine microchannel. Ind Eng Chem Res 51:5056-5066

26. Li XB, Li FC, Kinoshita H, Oishi M, Oshima M (2015) Dynamics of viscoelastic fluid droplet under very low interfacial tension in a serpentine T-junction microchannel. Microfluid Nanofluid 18: $1007-1021$

27. Wu Z, Wang L, Sundén B, Wadsö L (2016) Aqueous carbon nanotube nanofluids and their thermal performance in a helical heat exchanger. Appl Therm Eng 96:364-371

28. Fries DM, Waelchli S, von Rohr PR (2008) Gas-liquid twophase flow in meandering microchannels. Chem Eng J 135: S37-S45

29. Yagodnitsyna AA, Kovalev AV, Bilsky AV (2017) Experimental study of ionic liquid-water flow in T-shaped microchannels with different aspect ratios. J Phys Conf Ser 899:032026

30. Cao Z, Wu Z, Sattari-Najafabadi M, Sundén B (2017) Liquid-liquid flow patterns in microchannels. Proc. ASME 2017 Heat Transfer Conf., Paper no. HT2017-4729

31. Yao C, Liu Y, Xu C, Zhao S, Chen G (2018) Formation of liquidliquid slug flow in a microfluidic T-junction: effects of fluid properties and leakage flow. AICHE J 64:346-357 